\title{
eJRIEPS
}

Ejournal de la recherche sur l'intervention en éducation physique et sport

$31 \mid 2014$

Varia

\section{La Motivation en Situation. Une Revue de Questions en Education Physique}

Denis Pasco et David Spreux

\section{(2) OpenEdition}

Journals

Édition électronique

URL : http://journals.openedition.org/ejrieps/2304

DOI : 10.4000/ejrieps.2304

ISSN : 2105-0821

Éditeur

ELLIADD

\section{Référence électronique}

Denis Pasco et David Spreux, «La Motivation en Situation. Une Revue de Questions en Education Physique », eJRIEPS [En ligne], 31 | 2014, mis en ligne le 01 janvier 2014, consulté le 22 mars 2021. URL : http://journals.openedition.org/ejrieps/2304 ; DOI : https://doi.org/10.4000/ejrieps.2304

\section{(9) (1)}

La revue eJRIEPS est mise à disposition selon les termes de la Creative Commons Attribution 4.0 International License. 


\section{La Motivation en Situation. Une Revue de Questions en Education Physique}

\section{Denis Pasco* \& David Spreux*}

* Centre de recherche sur l'éducation, les apprentissages et la didactique, EA 3875, Université Européenne de Bretagne, France

** Faculté de psychologie et des sciences de l'éducation, Université de Mons, Belgique

\section{Résumé}

Un ensemble de théories cognitives de la motivation s'inscrivant dans le large domaine des études psychologiques sont aujourd'hui disponibles dans la littérature pour étudier, expliquer et interpréter la manière dont les sujets perçoivent et interprètent les activités dans lesquelles ils sont engagés en fonction de leurs besoins psychologiques. Au cours des dernières années, des chercheurs ont questionné l'idée de se centrer en éducation physique sur un tel domaine plutôt que d'investir des questions centrées sur les contenus spécifiques de l'éducation physique. Ils s'appuient sur une approche théorique de la motivation en situation basée sur le concept d'intérêt. L'objectif de cet article est de présenter une revue des questions adressées dans ce domaine de recherche, d'en dégager et critiquer les principaux résultats et, d'identifier des implications pour la pratique d'enseignement en éducation physique.

Mots-clés : intérêt en situation ; éducation physique ; apprentissage ; motivation ; revue de questions

La motivation consiste en une énergie et une direction. Elle a été avancée dans la littérature comme un facteur clé influençant la réussite (Wentzler \& Wigfried, 2009). En éducation physique $(E P)$, la motivation est un élément central à prendre en considération pour conduire les élèves à adopter et maintenir un style de vie sain par la pratique physique régulière. Un ensemble de théories cognitives de la motivation ont été proposées pour étudier et expliquer la manière dont les sujets perçoivent et interprètent les activités dans lesquelles ils sont engagés en fonction de leurs besoins psychologiques (Chen \& Ennis, 2009). La théorie des buts d'accomplissement, la théorie de l'efficacité personnelle ou la théorie des attentes et des valeurs constituent ainsi des théories explicatives des 
eJRIEPS 31 janvier 2014

effets de la motivation sur la réussite en contexte scolaire s'inscrivant dans le large domaine des études psychologiques (Cosnefroy, 2004).

Au cours de ces dernières années, des chercheurs (Chen, 2001; Chen \& Ennis, 2004 ; Chen, Martin, Sun \& Ennis, 2007) ont questionné l'idée de se centrer en EP sur un tel paradigme plutôt que d'investir des questions centrées sur les contenus spécifiques de I'EP. Ils argumentent en faveur de l'étude des rapports entre la motivation des élèves et les contenus spécifiques de l'EP. Ils s'appuient sur une approche théorique de la motivation en situation basée sur le concept d'intérêt. D'après Alexander et Jetton (1996), l'intérêt joue un rôle important dans le processus d'apprentissage et détermine en partie ce que nous choisissons d'apprendre et comment nous l'apprenons. Cette approche est originale et encore peu connue en France. Le but de cet article est de présenter une revue de questions des travaux menés dans ce domaine de recherche et de dégager des implications pour la pratique d'enseignement de l'EP.

\section{Construction théorique de l'intérêt en situation}

Krapp, Hidi et Renninger (1992) ont défini l'intérêt comme étant un état psychologique qui émerge de l'interaction d'une personne avec une activité. Dewey (1913) est le premier dans l'ouvrage "Interest and effort in education" à avoir investi théoriquement le concept d'intérêt. II ressort une distinction entre l'intérêt individuel et l'intérêt en situation.

L'intérêt individuel est caractérisé par les préférences d'un individu influencées par ses connaissances, ses croyances et ses valeurs. II s'agit d'un désir intrinsèque et stable de comprendre un sujet déterminé. L'intérêt individuel se développe au travers des interactions entre un individu et ses activités. D'après Schiefele, Krapp et Winteler (1992), l'intérêt individuel motive l'apprenant et conduit celui-ci à un apprentissage significatif. Lorsqu'un individu développe un intérêt pour un sujet déterminé, il identifie aisément les tâches d'apprentissage liées à ce sujet et en stocke à long terme les informations. Ce type d'intérêt influence le choix et la persévérance d'un apprenant dans l'apprentissage d'un contenu par rapport à un autre. Csikszentmihalyi (1990) compare l'intérêt individuel à un état de «flow » dans lequel un individu est complètement immergé dans une activité et fait l'expérience d'une pleine conscience de la tâche. L'intérêt individuel peut, par exemple, conduire un élève à choisir de suivre une classe d'histoire plutôt qu'une classe de mathématiques ou de préférer les sports individuels aux sports collectifs. II est également admis que l'influence sociale peut développer un intérêt individuel différent chez les garçons et les filles (Clifton \& Gill, 1994). Dans la mesure où l'intérêt individuel est 
eJRIEPS 31 janvier 2014

déterminé par des connaissances, des croyances et des valeurs personnelles, Hidi et Anderson (1992) soulignent l'extrême difficulté à concevoir un enseignement centré sur les intérêts individuels des élèves. Ils suggèrent plutôt de se centrer sur l'intérêt en situation.

L'intérêt en situation a été défini comme «l'effet attrayant des caractéristiques d'une activité sur les individus » (Chen, Ennis, Martin \& Sun, 2006, p.3). II est déterminé par les perceptions d'un apprenant par rapport aux caractéristiques d'une tâche. Murphy et Alexander (2000) ont montré qu'il était provoqué par les caractéristiques de l'environnement immédiat et n'avait pas forcément d'effet à long terme. Pour Reeve (1996), il résulte de l'interaction entre une activité donnée et une personne à un moment déterminé. De ce fait, soulignent Chen et Darst (2001), il serait plus aisé pour l'enseignant d'agir sur celui-ci pour motiver un apprenant.

Malgré cette distinction entre intérêt individuel et intérêt en situation, Hidi (2001) indique qu'ils constituent les deux facettes d'un même phénomène et s'influencent l'un et l'autre. Pour Hidi et Brendorff (1998), cette interaction favoriserait l'intériorisation progressive de l'intérêt en situation en intérêt individuel. Nous reviendrons plus loin sur la nature des rapports que l'on pourrait établir en EP entre ces deux facettes à partir des résultats des travaux menés dans ce domaine de recherche.

Deci (1992) rapproche le concept d'intérêt des motivations extrinsèque et intrinsèque caractérisées dans la théorie de l'auto-détermination (Deci \& Ryan, 1985). II montre que si l'intérêt est étroitement lié à la motivation intrinsèque, il peut aussi être associé à la motivation extrinsèque. Pour cet auteur, l'intérêt doit être envisagé dans la relation entre une personne et une activité. Lorsqu'une personne perçoit un intérêt, il/elle est engagée dans une activité. D'un point de vue théorique, l'intérêt résulte d'une mise en relation entre les besoins, désirs et capacités d'un individu avec les affordances d'une activité qui fournit une nouveauté, un défi ou un appel esthétique que l'individu recherche à un moment donné. Deci (1992) approche cette relation en identifiant sept dimensions réparties en trois catégories: les caractéristiques de l'activité, les dispositions mentales et l'expérience interactive.

Tout d'abord, les dimensions de la nouveauté et du défi sont incluses dans la catégorie des caractéristiques de l'activité. La nouveauté est présentée comme étant une information inconnue ou déficiente. Selon Spielberger et Starr (1994), il s'agit de la seule fonction capable d'engendrer un comportement d'exploration chez un individu. Le défi représente le niveau de difficulté que peut rencontrer un individu au regard de ses 


\section{eJRIEPS 31 janvier 2014}

compétences. Il est considéré par Harter (1978) comme un facteur suscitant l'engagement d'un individu dans une tâche ou une activité.

Ensuite, les dimensions «intention d'exploration », «éveil du désir» et « altération du temps » sont regroupées dans la catégorie des dispositions mentales. Le désir d'un individu de s'engager dans une activité est à l'origine du développement durable de l'intérêt que porte cet individu à cette activité. Les trois dimensions de la catégorie des dispositions mentales représentent la puissance de stimulation qui peut être observée dans des activités telles que les puzzles ou les jeux de réflexion (Mitchell, 1993). Pour Deci (1992), elles ont la capacité d'éveiller la perception de l'intérêt en situation d'un individu et peuvent augmenter sa motivation intrinsèque à s'engager dans une activité.

Enfin, les dimensions «demande d'attention» et «sens du plaisir» composent la catégorie de l'expérience interactive. L'expérience interactive d'une personne est la prise de conscience par un individu de son interaction avec une activité. La compréhension de cet intérêt est subjective. Pour Deci (1992), cette prise de conscience nécessite des qualités d'attention ou un sens du plaisir. Ces deux dimensions permettent à un individu d'apprécier le niveau de plaisir que lui offre une activité.

Chen (1996) est le premier auteur à avoir repris la construction théorique de l'intérêt en situation de Deci (1992) pour l'étudier en EP. Depuis cette première étude, il a investi de manière extensive avec ses associés ce domaine de recherche. Ces travaux complétés de ceux d'autres auteurs nous permettent aujourd'hui d'une part, de disposer de résultats empiriques sur l'intérêt en situation en EP et d'autre part, d'enrichir la construction théorique initiale de Deci (1992).

\section{Méthode pour l'identification et la sélection des articles supports}

Afin d'identifier les articles supports à la construction de cette revue de questions, nous avons interrogé les bases de données EBSCO Host (Academic Search Premier, Eric, Pascal, Psychology and Behavioral Sciences, Teacher Reference Center, Psycinfo), Science Direct et PubMed (recherche effectuée le 2 novembre 2012) avec les mots-clés "Situational interest" et "Physical education" appelés dans les résumés et titres des articles. Cette recherche a été complétée d'une interrogation du site Google Scholar en utilisant les mêmes mots clés.

Nous avons retenu les articles publiés en langue anglaise et expertisés par des pairs. Après suppression des doublons, nous disposions d'un ensemble de 16 articles. Enfin, nous avons adressé à Ang Chen la liste des 16 articles recensés et l'avons invité à la 


\section{eJRIEPS 31 janvier 2014}

compléter. II nous a adressé trois articles complémentaires (les auteurs remercient le Pr. Ang Chen d'avoir accepté de partager sa bibliographie personnelle). Au final, nous disposions de 19 articles supports à notre revue de questions. Le tableau 1 récapitule les différentes étapes d’identification et de sélection des articles.

Tableau I : Etapes d'identification et de sélection des articles

\begin{tabular}{|c|c|c|c|c|c|}
\hline Etape & Critères & $\begin{array}{c}\text { EBSCO } \\
\text { Host }\end{array}$ & $\begin{array}{c}\text { Science } \\
\text { Direct }\end{array}$ & PubMed & $\begin{array}{l}\text { Google } \\
\text { Scholar }\end{array}$ \\
\hline Identification des & Mots clés « Situational & & & & \\
\hline articles dans des & Interest » AND « Physical & & & & \\
\hline $\begin{array}{l}\text { bases de } \\
\text { données }\end{array}$ & $\begin{array}{l}\text { Education » appelés dans } \\
\text { le résumé et/ou le titre } \\
\text { - langue anglaise }\end{array}$ & 13 & 2 & 5 & 10 \\
\hline Sélection des & - expertise par des pairs & & & & \\
\hline $\begin{array}{l}\text { articles support } \\
\text { Sollicitation du } \mathrm{Pr}\end{array}$ & - suppression des doublons & 5 & 0 & 2 & 9 \\
\hline Ang Chen & Bibliographie personnelle & \multicolumn{4}{|c|}{3 articles } \\
\hline Total & & \multicolumn{4}{|c|}{19 articles } \\
\hline
\end{tabular}

Les 19 articles ont été lus à plusieurs reprises puis catégorisés en fonction des questions de recherche adressées par les auteurs. Cette catégorisation a abouti à identifier cinq questions principales (1) Quelles sont les dimensions qui composent le construit de l'intérêt en situation en éducation physique et comment peut-on les mesurer ? (2) Comment s'établissent les rapports entre les différentes dimensions de l'intérêt en situation ? (3) Qu'est-ce qui influence l'intérêt en situation des élèves ? (4) L'intérêt en situation affecte-t-il l'apprentissage des élèves ? et (5) Comment s'établissent les rapports entre intérêt en situation et intérêt individuel ?

Nous détaillons ci-dessous les études conduites pour répondre à chacune de ces questions.

\section{Quelles sont les dimensions qui composent le construit de l'intérêt en situation en éducation physique et comment peut-on les mesurer ?}

L'objectif de l'étude de Chen, Darst et Pangrazi (1999) était d'explorer le construit de l'intérêt en situation en EP et d'en valider un instrument de mesure. Posant que l'intérêt en situation se caractérise par une subjectivité personnelle, les auteurs ont considéré que les mesures d'intérêt en situation ne pouvaient être recueillies que lorsque les participants étaient placés dans une situation dans laquelle ils devaient différencier deux ou plusieurs activités comparables en fonction des caractéristiques attribuées à ces activités. L'étude 
eJRIEPS 31 janvier 2014

de Chen et al. (1999) a été menée en quatre étapes indépendantes en termes de sujets, de recueil de données et d'analyse de données. Chaque étape a servi de fondation aux investigations conduites dans l'étape suivante.

L'objectif de la première étape était de préparer des énoncés représentant les différentes dimensions du construit théorique. Cent-quatre vingt neuf élèves du secondaire ont visionné deux vidéos, l'une présentant des acrobaties en gymnastique sportive et l'autre une activité de course à pied. Ces deux activités ont été choisies par les chercheurs, en posant l'hypothèse que les acrobaties en gymnastique présentaient un intérêt élevé pour les élèves du secondaire tandis que la course à pied constituait un intérêt faible pour eux. Après avoir visionné chaque vidéo, les élèves ont évalué leurs sentiments sur l'activité à l'aide de 68 énoncés rédigés par les auteurs de l'étude et représentant les différentes dimensions supposées du construit théorique de l'intérêt en situation. Les réponses des élèves ont été analysées pour déterminer les énoncés permettant de distinguer les sentiments des élèves vis-à-vis des deux activités. Quarante-trois énoncés et quatre énoncés sur l'intérêt total ont ainsi été sélectionnés pour être utilisés dans l'étape suivante.

Dans cette seconde étape, 201 élèves du secondaire ont évalué l'intérêt qu'ils accordaient aux deux activités présentées dans les vidéos à l'aide des énoncés sélectionnés à l'étape précédente. Une analyse factorielle exploratoire a été conduite sur les réponses des élèves concernant la vidéo de gymnastique de manière à valider les mesures multidimensionnelles de l'intérêt en situation. Cette analyse a révélé que cinq des sept dimensions du modèle de Deci (1992) étaient observables dans le domaine de l'EP. Les dimensions de désir et d'altération du temps n'ont pas été observées comme dimensions indépendantes tandis que la dimension "sens du plaisir " a été renommée en plaisir instantané dans la mesure où le plaisir instantané a été défini comme étant un processus qui voit émerger le sentiment d'intérêt observé dans cette étude (Harackiewicz, Sansone \& Manderlink, 1985). Vingt énoncés (quatre pour chacune des cinq dimensions) ont été retenus à l'issue de cette étape et distribués de manière aléatoire pour constituer le questionnaire de l'intérêt en situation. Chaque énoncé est accompagné d'une échelle de type Likert en cinq points ( 5 = tout à fait d'accord, 1 = tout à fait en désaccord). Ces 20 énoncés sont complétés de quatre énoncés mesurant l'intérêt total.

L'objectif de la troisième étape était de confirmer l'analyse factorielle exploratoire de l'étape précédente. Quarante-neuf élèves du secondaire ont complété le questionnaire de l'intérêt en situation directement après avoir visionné chacune des vidéos précédentes. 


\section{eJRIEPS 31 janvier 2014}

L'analyse factorielle confirmatoire a confirmé la structure du construit établi à l'étape précédente et validé les 24 énoncés du questionnaire.

La quatrième étape consistait à réexaminer le construit de l'intérêt en situation identifié dans les étapes précédentes dans le cadre de tâches d'apprentissage en EP. II s'agissait donc de répliquer les analyses faites au cours des étapes précédentes afin de déterminer si le construit de l'intérêt en situation pouvait être maintenu lorsque les élèves participaient à des activités physiques. Cent-quatre vingt onze élèves du secondaire ont participé à cette étape. Ils ont participé à deux tâches motrices. La première tâche consistait en une série de passes à hauteur de la poitrine par deux en face à face à une distance de quatre mètres et demi. Cette première tâche a été identifiée par les chercheurs comme ayant un intérêt faible pour les élèves. La seconde tâche consistait en une circulation définie de la balle et une rotation des joueurs dans des positions définies autour de la raquette se concluant par un tir. Cette tâche était considérée par les chercheurs comme comportant un intérêt élevé pour les élèves. Après chaque activité, les élèves ont complété l'échelle de mesure de l'intérêt en situation. L'analyse factorielle exploratoire de ces données a révélé une solution en cinq facteurs expliquant $66.80 \%$ de la variance. Cette solution a servi de base à l'analyse factorielle confirmatoire. Les résultats ont validé une structure du construit en cinq dimensions avec des coefficients de Cronbach de respectivement .78 pour la dimension de la nouveauté, .80 pour celle du défi, .90 pour la demande d'attention, .91 pour l'intention d'exploration et .90 pour le plaisir instantané. De plus, un coefficient de .95 a été obtenu pour la mesure de l'intérêt total. Ces résultats fournissent une évidence supportant que l'intérêt en situation se caractérise par un construit observable en cinq dimensions dans la participation à des tâches motrices en EP.

Ce construit a été confirmé dans l'enseignement primaire (Sun, Chen, Ennis, Martin \& Shen, 2008). L'intérêt individuel dans l'activité physique est mesuré dans les études à l'aide d'un questionnaire (Chen \& Darst, 2001). Au début du questionnaire, il est demandé aux participants d'identifier une activité à l'école ou hors de l'école qu'ils trouvent très intéressante et à laquelle ils attribuent sept sur une échelle de type Likert en sept points (1=pas du tout intéressante ; $7=$ très intéressante). On leur demande ensuite de classer sur l'échelle en sept points un groupe d'activités physiques en comparaison avec l'activité qu'ils ont identifiée comme très intéressante pour eux. Ce groupe d'activités comprend des activités individuelles (athlétisme, natation), des activités collectives (football, basket-ball), des activités de forme/santé (musculation, step), des activités artistiques (danse, gymnastique rythmique) ou encore des activités de pleine nature (escalade, vélo tout 
eJRIEPS 31 janvier 2014

terrain). Selon Tobias (1994), ce contexte de mesure peut minimiser la possibilité que les répondants exercent leur propre interprétation du classement des activités du groupe et maintient ainsi la validité interne de la mesure.

\section{Comment s'établissent les rapports entre les différentes dimensions de l'intérêt en situation?}

Après avoir validé un contruit théorique de l'intérêt en situation reposant sur cinq dimensions, Chen, Darst et Pangrazi (2001) ont examiné l'influence de chacune des dimensions sur l'intérêt en situation ainsi que l'influence des différentes dimensions entre elles. Pour ce faire, les auteurs ont conduit une étude auprès de 472 élèves de collège (51\% de garçons; $49 \%$ de filles) représentant de manière équilibrée les classes de cinquième ( $\mathrm{M}$ âge $=12.76, \sigma=.54)$, quatrième ( $\mathrm{M}$ âge $=13.82, \sigma=.57$ ) et troisième $(\mathrm{M}$ âge $=14.94, \sigma=.51$ ). Les élèves ont été séparés en deux groupes et confrontés à deux tâches différentes, l'une à faible intérêt et l'autre à fort intérêt, de manière à préserver la validité interne de l'intérêt en situation. Les 281 élèves (57\% de garçons ; 43\% de filles) du premier groupe ont observé des vidéo de course à pied et d'acrobaties gymniques en dehors de leur classe d'EP. Les élèves de l'autre groupe ( $\mathrm{N}=191 ; 47 \%$ garçons ; 53\% de filles) ont participé durant leur cours d'EP aux tâches de passes à la poitrine et de passes et tir au basket-ball décrites précédemment. Immédiatement après les tâches, les élèves ont complété le questionnaire d'intérêt en situation.

Les résultats ont montré que les élèves évaluaient les acrobaties gymniques et les passes et tir au basket-ball comme ayant un plus grand intérêt en situation que la course à pied et les passes à la poitrine du basket-ball. Les coefficients de corrélation obtenus pour les acrobaties en gymnastique et les passes et tir en basket-ball ont montré une forte corrélation entre le plaisir instantané et l'intérêt total. Autrement dit, l'intérêt total est fortement corrélé avec le sentiment de plaisir généré par ces activités. Le plaisir instantané ressort comme la source qui influence le plus le construit de l'intérêt en situation. Cette dimension joue un rôle relativement indépendant dans le modèle. L'intention d'exploration a aussi une influence significative sur l'intérêt total. Par contre, la dimension « défi » a un impact négatif sur l'intérêt total suggérant qu'un plus haut niveau de défi pourrait conduire à un plus faible intérêt en situation. Les dimensions « nouveauté » et « demande d'attention » n'ont pas d'impact significatif sur l'intérêt total. En ajustant le modèle de manière à identifier les relations entre les différentes dimensions 


\section{eJRIEPS 31 janvier 2014}

du construit lorsque les élèves pratiquent des tâches à fort intérêt, Chen et al. (2001) ont abouti au modèle présenté à la figure 1 .

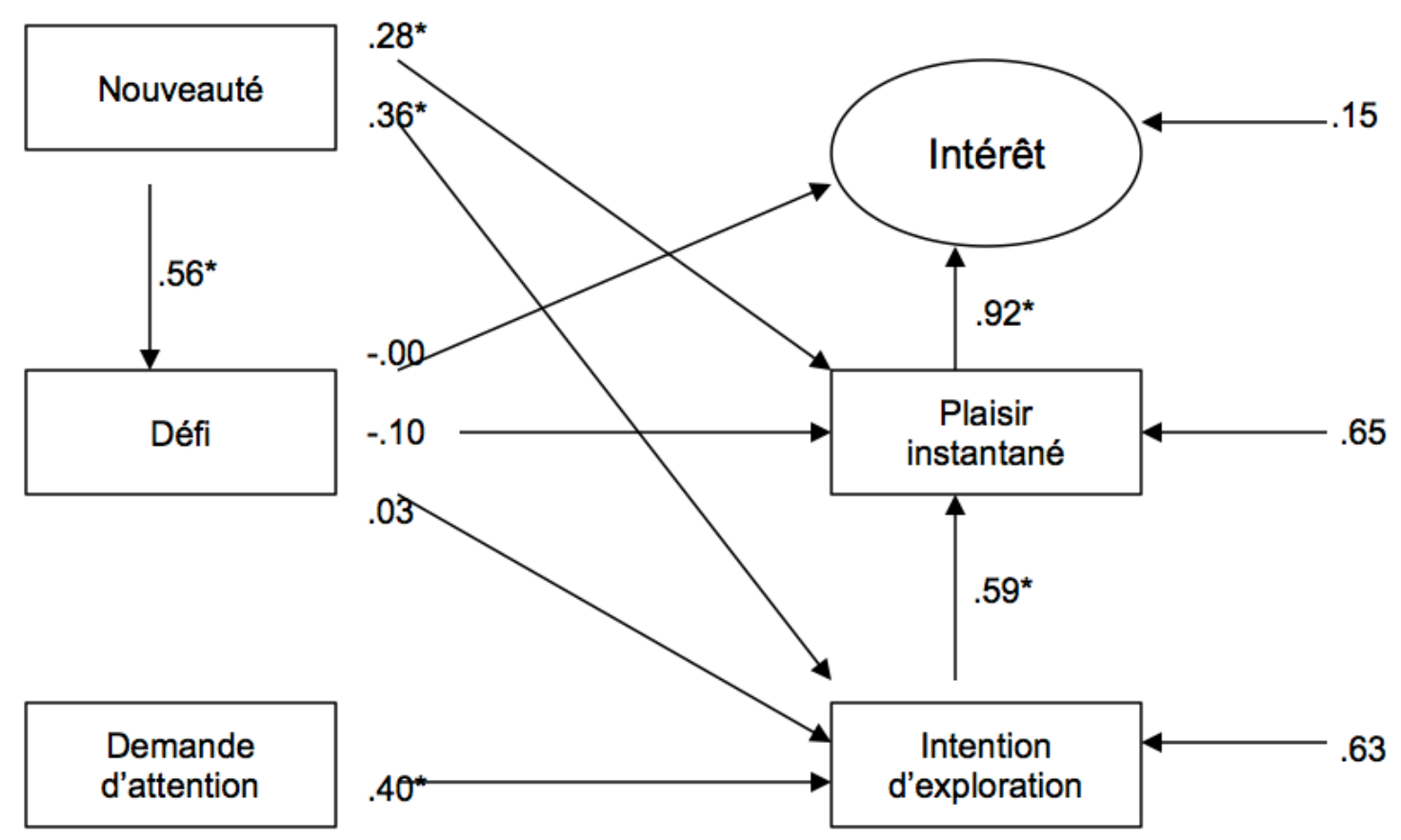

*significatif $(p=01)$

Figure 1. Modèle de l'intérêt en situation en éducation physique

Ce modèle confirme que le plaisir instantané est la dimension présentant le plus d'influence. L'intention d'exploration est identifiée comme la seconde dimension la plus importante. Elle influence les effets venant de la nouveauté et de la demande d'attention sur le plaisir instantané. La dimension « défi » est une dimension isolée dans le modèle. Elle a un très faible effet sur l'intérêt total, le plaisir instantané et l'intention d'exploration. De plus, cette dimension absorbe le plus large effet venant de la nouveauté. En comparaison avec le modèle produit lorsque les élèves observent des activités à fort intérêt (acrobaties en gymnastique), le plaisir instantané est relié de manière plus directe avec l'intérêt total. De plus, l'effet de la nouveauté est divisé et l'effet du défi diminué. Ces différences suggèrent que le défi est susceptible de contribuer à l'intérêt total lorsque les élèves observent des tâches présentant de l'intérêt alors que lorsqu'ils les pratiquent, le défi n'y contribue pas du tout. II ne conduit pas non plus dans la pratique au désir d'explorer la tâche et au plaisir instantané.

L'étude de Chen et al. (2001) a ainsi permis de disposer d'un modèle de l'intérêt en situation en éducation physique comprenant les effets des différentes dimensions. Si ce 
eJRIEPS 31 janvier 2014

modèle est aujourd'hui mobilisé dans un ensemble d'études en éducation physique, il n'est pas sans poser de questions. En effet, si la dimension de défi ressort comme une dimension isolée ayant si peu d'impact sur l'intérêt total, le plaisir immédiat, l'intention d'exploration et aucun impact sur la demande d'attention et la nouveauté dans le cadre de tâches d'activité physique, il est légitime de s'interroger sur la maintien de cette dimension en EP. De plus, la demande d'attention ayant un effet indirect sur l'intérêt en situation à travers l'intention d'exploration et aucun effet sur les autres dimensions, il faudrait s'interroger sur l'hypothèse de construire une dimension unique traduisant une exploration de la tâche appuyée sur une demande d'attention initiale. Enfin, il ressort que si l'intérêt et le plaisir sont deux facteurs reliés de la motivation en situation, ils constituent deux dimensions séparées renvoyant peut-être à des construits différents. Des études complémentaires sont nécessaires à tous les niveaux d'enseignement de manière à éprouver la robustesse de ce modèle explicatif de la motivation en situation en EP.

\section{Qu'est-ce qui influence l'intérêt en situation des élèves ?}

5.1. Facteurs individuels : le sexe et les habiletés motrices

Le sexe et le niveau d'habileté motrice ont été avancés dans la littérature comme susceptibles d'affecter la motivation des élèves en éducation physique. En effet, Griffin (1985) a montré que la perception qu'avait un apprenant de son niveau d'habileté motrice influençait sa motivation à s'engager dans une tâche en éducation physique. De même, Lee, Fredenburg, Belcher et Cleveland (1999) ont montré qu'un appenant était d'autant plus motivé par une tâche qu'il la considérait comme adaptée à son sexe. Des études ont dégagé que le sexe de l'apprenant et son niveau de compétence avaient un impact sur l'intérêt individuel et l'intérêt en situation en mathématiques (Mathew, 1993), sciences (Baumert, 1998 ; Gardner, 1998) et lecture (Anderson, Shirey, Wilson \& Fieldings, 1987).

L'étude de Chen et Darst (2002) visait à étudier l'influence du sexe et du niveau d'habileté des élèves sur leur intérêt individuel et leur intérêt en situation en éducation physique. Cent-quatre vingt onze élèves de classe de quatrième ( $\mathrm{M}$ âge $=12.76, \sigma=.54$ ) et de troisième $(M$ âge $=13.81, \sigma=.58)$ de collège ont participé à cette étude $(53 \%$ de garçons, $47 \%$ de filles). Les auteurs ont examiné dans quelle mesure l'intérêt individuel et l'intérêt en situation des élèves variaient en fonction du sexe et de leur niveau d'habileté motrice dans les deux tâches de basket-ball décrites précédemment : passes-poitrine et circulation de la balle et des joueurs autour de la zone finalisée par un tir. L'échelle de mesure de l'intérêt en situation a été utilisée pour évaluer l'intérêt en situation des élèves 
eJRIEPS 31 janvier 2014

immédiatement après chaque tâche. L'intérêt individuel a été mesuré à l'aide du questionnaire développé par Chen et Darst (2001). Le niveau d'habileté motrice des élèves en Basketball a été déterminé à l'aide des tests standardisés de l'association américaine pour la santé, l'éducation physique, le loisir et la danse.

Les résultats montrent des différences significatives entre les garçons et les filles dans leur intérêt en situation pour la tâche de circulation de la balle et des joueurs autour de la zone ponctuée par un tir. Pour autant, les auteurs refusent de conclure que ces différences seraient liées au sexe des élèves. En effet, en croisant le niveau d'habileté des élèves et leur sexe sur la tâche où des différences entre garçons et filles sont apparues, Chen et Darst (2002) ont regroupé les élèves en deux groupes distincts, l'un représentant le quartile d'intérêt élevé en situation $(N=51)$ et l'autre le quartile de l'intérêt faible en situation $(N=48)$. Une fois ces deux groupes constitués, les résultats ont révélé qu'ils différaient dans leur composition selon les niveaux d'habileté des élèves $(p=.93)$, leur niveau d'intérêt individuel $(p=.02)$ mais pas leur sexe $(p=.93)$. Chen et Darst (2002) confirment ainsi que les différences de sexe dans l'intérêt en situation pourraient être attribuables à des différences de niveaux d'habileté. Les filles se situant majoritairement au niveau initial d'apprentissage privilégieraient l'intérêt en situation comme principal facteur de motivation tandis que pour les garçons se situant à des niveaux intermédiaires d'apprentissage, l'intérêt individuel remplacerait l'intérêt en situation comme principal facteur de motivation.

Chen et Darst (2001) n'avaient pas trouvé de corrélation significative entre le niveau d'habileté des élèves dans une tâche d'apprentissage au basket-ball et l'intérêt en situation rapporté dans cette tâche. Shen, Chen, Tolley et Scrabis (2003) n'ont pas constaté de différence significative dans l'intérêt en situation rapporté par des garçons et des filles de collège $(\mathrm{N}=57)$ dans une activité de danse.

5.2. Facteurs contextuels : la conception de la tâche

D'après Mitchell (1993), l'intérêt en situation résulte de la reconnaissance par des pratiquants d'une ou plusieurs caractéristiques attrayantes d'une tâche d'apprentissage spécifique. La manière dont un enseignant d'éducation physique concevrait une tâche d'apprentissage en éducation physique pourrait donc être un élément important dans le but de provoquer un intérêt en situation de la part des élèves.

Chen et Darst (2001) ont testé cette hypothèse en étudiant l'influence de la conception des tâches sur l'intérêt en situation des élèves en éducation physique. Dans la mesure où les dimensions physiques et cognitives représentent deux composants fondamentaux 


\section{eJRIEPS 31 janvier 2014}

impliqués dans les activités physiques, Chen et Darst (2001) ont examiné dans quelle mesure les demandes physiques et cognitives des tâches d'apprentissage en éducation physique influençaient l'intérêt en situation des élèves.

Deux-cent quatre vingt douze élèves de collège (51\% de garçons, $49 \%$ de filles) ont participé à cette étude (72 en classe de cinquième, 99 en quatrième et 71 en troisième). Quatre tâches ont été proposées aux élèves prenant en compte les dimensions physiques et cognitives des tâches d'apprentissage en basket-ball : (a) une tâche d'analyse à l'aide d'une grille d'observation de la diversité des habiletés utilisées par des joueurs et joueuses de haut-niveau dans une vidéo de cinq minutes considérée par les auteurs comme présentant une dimension cognitive élevée mais aucune dimension physique, (b) une tâche de passes à hauteur de la poitrine en position stationnaire entre deux élèves séparés d'environ quatre mètres considérée comme présentant une exigence cognitive et physique faible pour ces élèves, (c) une tâche de courses à but défensif considérée comme présentant une dimension cognitive faible et une dimension physique élevée et, (d) une tâche de circulation définie de la balle et de rotation des joueurs dans des positions définies autour de la raquette se concluant par un tir considérée comme présentant une dimension cognitive et physique importante. Directement après avoir pratiqué ces tâches, les élèves ont renseigné le questionnaire de l'intérêt en situation (Chen et al., 1999).

Les résultats indiquent que la conception de la tâche a un effet significatif sur l'intérêt en situation. Ils montrent que les deux tâches présentant une demande cognitive (analyse de vidéo et passes-tir) reçoivent les scores les plus hauts dans toutes les dimensions. Les analyses statistiques n'indiquent aucune différence significative dans les scores de ces deux tâches sur les différentes dimensions. La tâche de passes-poitrine recueille les scores les plus bas sur chaque dimension suivie de la tâche de courses à but défensif. La demande cognitive de la tâche ressort comme le facteur déterminant de l'intérêt en situation. Ce résultat n'est pas sans interroger la nature des apprentissages en EP. Si l'intérêt en situation est déterminé principalement par la demande cognitive de la tâche, les enseignants d'EP devraient réduire ou éliminer la demande physique des tâches d'apprentissage. D’ailleurs, les résultats de cette étude sont consistants avec les résultats obtenus sur l'intérêt en situation dans des tâches de lecture (Hidi \& Baird, 1988). Chen et Darst (2001) réfutent cette conclusion en montrant qu'il est très clair dans leurs données qu'un haut niveau de demande physique d'une tâche d'apprentissage est très intéressant pour la motivation en situation si la tâche comporte aussi une forte demande cognitive. 


\section{eJRIEPS 31 janvier 2014}

Chen et al. (2006) ont comparé les effets d'un programme d'éducation physique et à la santé avec ceux d'un programme traditionnel auprès de 7000 élèves. Les auteurs ont conclu que l'intérêt en situation était une dimension qui devait être prise en considération dans la conception d'un programme en éducation physique.

\section{L’intérêt en situation affecte-t-il l'apprentissage des élèves ?}

L'apprentissage en EP conduit à deux types de résultats: des connaissances de type cognitif et des habiletés motrices (Shen, Chen \& Guan, 2007). Ces résultats sont observables à travers des comportements. Ils indiquent dans quelle mesure un apprenant s'est engagé et a appris dans la tâche (Chen \& Ennis, 2009). Les études développées précédemment sont de nature descriptive de sorte que les résultats ne permettent pas d'établir de lien direct entre l'intérêt en situation, les comportements motivés des élèves et l'apprentissage en éducation physique. Les études ci-dessous ont cherché à identifier si l'intérêt en situation des élèves pouvait être relié à leurs apprentissages en EP.

Chen, Shen, Scrabis et Tolley (2002) ont examiné la relation entre l'intérêt en situation et les résultats d'apprentissage auprès d'un échantillon d'élèves de collège $(\mathrm{N}=104)$ engagés dans six cycles différents d'activité physique durant 17 semaines. L'engagement a été apprécié par l'intensité physiologique mesurée à l'aide d'un instrument de mesure de type pédomètre et validée à partir des résultats obtenus par des mesures de fréquence cardiaque. L'apprentissage a été apprécié par des tests de connaissances et d'habiletés après chaque cycle d'activité physique. Les résultats ont montré que l'intérêt individuel des élèves avait une relation significative positive mais faible avec l'intensité physiologique $(r=$ $.35, p<.01)$ et la réussite $(r=.24, p<.01)$ tandis que l'intérêt en situation avait une forte relation positive significative avec l'intensité physiologique $(r=.67, p<.01)$. Des analyses complémentaires ont confirmé la faible prédiction de l'intérêt individuel concernant les deux résultats d'apprentissage mesurés et la forte prédiction de l'intérêt en situation concernant l'intensité physiologique. Autrement dit, lorsque les élèves identifient un intérêt en situation dans une tâche d'apprentissage, ils s'y engagent physiquement plus intensément.

Ces mêmes auteurs ont poursuivi leurs investigations en examinant les relations entre l'intérêt individuel, l'intensité physique, l'intérêt en situation et les résultats d'apprentissage dans un cycle de danse auprès de 600 élèves de collèges (Shen, Chen, Tolley \& Scrabis, 2003). Les résultats d'apprentissage ont été mesurés à travers l'intensité de l'activité physique et des évaluations des connaissances et des habiletés en danse. Les résultats 


\section{eJRIEPS 31 janvier 2014}

ont montré que les filles avaient un intérêt individuel plus fort pour la danse que les garçons. La corrélation entre l'intérêt individuel et les résultats d'apprentissage était plus forte pour les filles $(r=.62, p<.01)$ que pour les garçons $(r=.26, p>.05)$. Cependant, les garçons et les filles ont tous les deux évalué les tâches d'apprentissage proposées en danse comme ayant de l'intérêt en situation. Pour autant, Shen et al. (2003) n'ont trouvé aucune relation significative entre l'intérêt en situation et les résultats d'apprentissage des élèves. Pour les auteurs, l'intérêt en situation des garçons semble supplanter leur faible intérêt individuel pour la danse.

L'étude de Zhu, Chen, Ennis, Sun, Hopple, Bonello, Bae et Kim (2009) a aussi adressé la question de la relation entre l'intérêt en situation, l'engagement cognitif et la réussite des élèves en EP. Les auteurs ont examiné les résultats de 670 élèves de deuxième année de cours élémentaire à l'issue d'un programme d'éducation physique et à la santé comprenant 30 leçons. L'engagement cognitif a été apprécié à travers les réponses des élèves contenues dans un journal de bord accompagnant les leçons tandis que la réussite a été évaluée à travers le gain de connaissances entre les résultats à un test de connaissances administré avant et après le programme. Les résultats n'ont pas permis aux auteurs d'établir de relation significative entre l'intérêt en situation et la réussite des élèves et, entre l'intérêt en situation et les résultats du journal de bord.

Les auteurs dans ce domaine de recherche ne sont donc pas parvenus à établir un lien direct entre l'intérêt en situation et les résultats d'apprentissage des élèves en EP. Au contraire de l'intérêt individuel qui apparaît établi dans le temps, l'intérêt en situation est immédiat et provoque un engagement de courte durée. Selon Hidi et Harackiewicz (2000), cet engagement est suffisant pour engager les élèves vers le processus d'apprentissage. Les études présentées ci-dessus révèlent qu'il n'est pas suffisant pour maintenir les élèves dans le processus d'apprentissage de manière à ce qu'ils construisent des apprentissages en EP. II ressort aussi de ces études que les élèves associent avant tout à l'EP une pratique physique et que l'apprentissage de type cognitif ne peut se substituer, pour les élèves, à la pratique physique. Le rôle de l'intérêt en situation ressort dans les réponses des élèves comme fortement dépendant de la tâche d'apprentissage.

\section{Comment s'établissent les rapports entre intérêt en situation et intérêt individuel?}

Les études présentées ci-dessus révèlent que les rapports entre intérêt individuel et intérêt en situation en EP sont complexes. En partant des travaux dégageant une relation significative entre le niveau de pratique des élèves, leur intérêt individuel et leur intérêt en 


\section{eJRIEPS 31 janvier 2014}

situation (Chen \& Darst, 2001 ; Chen, Shen, Tolley \& Scrabis, 2002), Chen et Hancock (2006) ont produit un modèle théorique intégré de la motivation en activité physique des adolescents et de leur changement de comportements vis-à-vis de la pratique physique (figure 2).

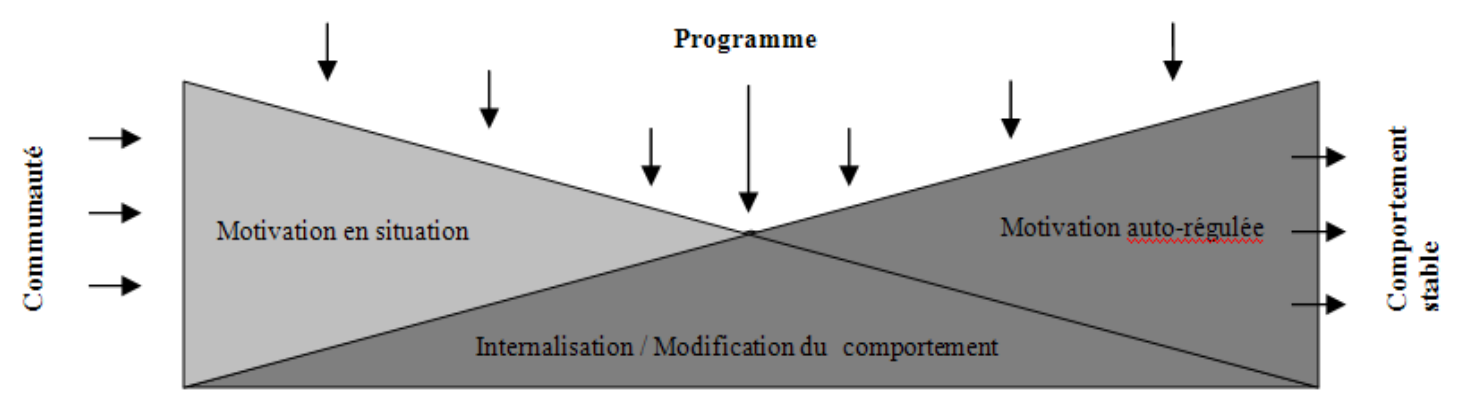

Niveaux de changement de comportement

\begin{tabular}{|c|c|c|c|}
\hline $\begin{array}{l}\text { Variables } \\
\text { personnelles }\end{array}$ & Acclimatation & Compétence & Efficacité \\
\hline $\begin{array}{l}\text { Connaissances } \\
\text { \&compétences }\end{array}$ & Faibles & Développées & Suffisantes \\
\hline Conceptions & Erronées & Précises & $\begin{array}{l}\text { Très } \\
\text { précises }\end{array}$ \\
\hline $\begin{array}{l}\text { Sources de } \\
\text { motivation }\end{array}$ & $\begin{array}{l}\text { Intérêt en } \\
\text { situation }\end{array}$ & $\begin{array}{l}\text { Intérêt en } \\
\text { situation et } \\
\text { individuel }\end{array}$ & $\begin{array}{l}\text { Auto- } \\
\text { régulé }\end{array}$ \\
\hline$\frac{\text { Comportement }}{\text { attendu }}$ & Aucun & Apparaissant & Stabilisé \\
\hline
\end{tabular}

Figure 2. Modèle théorique de la motivation en activité physique et du changement de comportement.

Dans ce modèle, la motivation des adolescents pour l'activité physique et leur changement de comportement sont rapportés à trois niveaux de pratique en activité physique: acclimatation, compétence et efficacité. Suivant leur niveau de pratique initial dans une activité physique, les intérêts des adolescents dans les tâches d'apprentissage en éducation physique varient. Ainsi, au niveau «acclimatation ", les adolescents disposant de connaissances et d'habiletés motrices limitées dans l'activité s'engagent dans l'activité à partir d'un intérêt en situation. Cet engagement est en lien avec le statut de l'activité physique dans le milieu social dans lequel ils évoluent. Ces adolescents sont sensibles à des motivateurs inscrits dans le contexte pour apprendre des connaissances et développer des habiletés. Le programme devrait être centré à ce stade sur des tâches d'apprentissage provoquant un fort intérêt en situation. 


\section{eJRIEPS 31 janvier 2014}

Au niveau « compétence », les adolescents commencent à développer les connaissances et les habiletés motrices pour le développement des comportements attendus. Ces connaissances et ces habiletés sont très utiles pour permettre à l'adolescent de développer des croyances en son efficacité personnelle. A ce stage, les jeunes commencent à développer une motivation auto-régulée provenant d'une intériorisation de la motivation en situation. Par exemple, ils peuvent commencer à réaliser que l'activité physique n'est pas uniquement un plaisir immédiat (motivation en situation) mais qu'elle s'accompagne de bénéfices pour la santé (motivation auto-régulée basée sur la valeur de l'activité physique). Ce niveau de pratique constitue une étape fondamentale dans le processus conduisant un adolescent à adopter des comportements auto-régulés d'engagement à long terme dans l'activité physique. Les tâches d'apprentissage du programme devraient à ce stade être conçues dans le but de convaincre les adolescents que les connaissances et habiletés qu'ils apprennent pourront les aider dans leur vie physique future.

Enfin, au niveau « efficacité », les adolescents ont acquis suffisamment de connaissances et d'habiletés motrices pour comprendre et intérioriser la valeur de l'engagement dans l'activité physique. L'adolescent fait preuve d'une motivation auto-régulée le conduisant à un engagement stable dans la pratique physique. Cet engagement peut éventuellement devenir durable. L'adolescent devient alors capable de modifier son comportement lorsqu'il apprend de nouvelles connaissances et de nouvelles habiletés. Les tâches d'apprentissage du programme devraient à ce stade être centrées sur des stratégies de gestion de sa santé à travers la pratique physique.

Le modèle théorique de Chen et Hancock (2006) a été testé dans les études de Shen et Chen (2006, 2007). Shen et Chen (2006) l'ont mis à l'épreuve en étudiant les relations entre l'intérêt individuel et l'intérêt en situation durant un cycle de volley-ball avec 80 élèves de sixième débutants (43 garçons ; 37 filles). Les résultats obtenus confirment qu'au fur et à mesure que ces élèves débutants apprennent des connaissances et des habiletés motrices, l'intérêt en situation manifesté initialement dans les tâches d'apprentissage est progressivement intériorisé en intérêt individuel. Les auteurs constatent qu'un fort intérêt en situation dans une tâche d'apprentissage peut contrebalancer un intérêt individuel faible et jouer un rôle important dans l'engagement cognitif des élèves. L'intérêt en situation initial aurait donc un impact très fort sur l'intérêt individuel futur des élèves débutants. Ces résultats ont été confirmés par Shen et Chen (2007) dans le cadre d'un cycle de softball avec 177 élèves de sixième. 


\section{eJRIEPS 31 janvier 2014}

\section{Implications pour la pratique}

Alors que les enseignants peuvent parfois se sentir dépossédés de leur rôle de concepteur de tâches d'apprentissage à l'occasion de la mise en œuvre de nouveaux programmes rédigés par des experts au niveau national, les résultats présentés dans cette revue de littérature suggèrent que l'intérêt en situation est directement influencé par la nature des tâches d'apprentissage, affirmant ainsi le rôle central de l'enseignant dans la conception de tâches pour promouvoir la motivation des élèves. Couplés avec les résultats montrant que la disposition mentale motivationnelle (e.g., théorie des buts d'accomplissement) pourrait avoir une influence limitée sur les comportements d'apprentissage (Chen, 2001), les résultats présentés dans cette revue invitent les chercheurs à lier la motivation avec le contenu de l'apprentissage dans le cadre du contexte culturel dans lequel se développe l'enseignement de l'éducation physique considéré.

Cinq dimensions ont été identifiées comme susceptibles d'affecter la motivation en situation des élèves : la nouveauté, le défi, l'intention d'exploration, la demande d'attention et le plaisir instantané. Elles constituent autant de variables que l'enseignant peut manipuler directement dans sa conception de tâches d'apprentissage. Cependant, les résultats montrent que la demande cognitive de la tâche est un facteur déterminant de l'intérêt en situation. Ainsi, pour promouvoir l'intérêt en situation des élèves, l'enseignant devrait s'attacher en premier lieu à la demande cognitive des tâches. Il pourrait ainsi, par exemple dans les sports collectifs, concevoir des tâches nécessitant peu de déplacements de joueurs mais des prises d'informations complexes du porteur de balle sur plusieurs partenaires et plusieurs adversaires. Pour autant, il ressort aussi des études que les élèves associent avant tout à l'EP une pratique physique et que l'apprentissage de type cognitif ne pourrait se substituer, pour les élèves, à la pratique physique. L'enjeu pour l'enseignant d'EP devient alors d'inclure dans les tâches d'apprentissage des demandes cognitives élevées et des demandes physiques élevées. Pour reprendre l'exemple cidessus, il ajoutera alors à sa tâche des déplacements et des circulations des joueurs à des rythmes de courses variés.

A ceci s'ajoute une troisième dimension. En effet, l'examen des différentes relations entre les dimensions du construit de l'intérêt en situation ont montré que le plaisir immédiat et l'intention d'exploration constituaient les deux dimensions présentant le plus d'influence sur l'intérêt en situation des élèves. A partir de ces résultats, il faudrait encourager les enseignants d'EP à concevoir des tâches à forte demande cognitive et physique 


\section{eJRIEPS 31 janvier 2014}

suscistant chez les élèves un plaisir instantané et une intention d'exploration, c'est-à-dire la volonté de poursuivre son engagement pour en découvrir plus sur les différentes facettes de la tâche d'apprentissage.

Enfin, certains des résultats présentés dans cette revue indiquent que l'effet de la tâche sur la motivation en situation est peu influencé par les niveaux d'habileté. Ce résultat pourrait suggérer que, lorsqu'ils construisent leurs tâches, les enseignants devraient se centrer sur la tâche elle-même. Les élèves semblent être attirés avant tout par un haut niveau d'intérêt en situation et ceci indépendamment de leurs niveaux d'habileté. Si l'on suit les résultats présentés dans cette étude, ce haut niveau d'intérêt en situation est susceptible de provoquer un engagement des élèves dans le processus d'apprentissage débouchant sur des apprentissages affectant à terme l'intérêt individuel des élèves pour les activités physiques. Pour reprendre l'expression de Subramaniam (2009), ce processus mobilise « la puissance de l'intérêt » pour engager durablement les élèves dans l'activité physique.

\section{Bibliographie}

Alexander, P.A., \& Jetton T.L. (1996). The role of importance and interest in the processing of text. Educational Psychology Review, 8, 89-121.

Anderson, R.C., Shirey, L.L., Wilson, P.T., \& Fieldings, L.G. (1987). Interestingness of children's reading material. In R.E Snow, \& M.J. Farr (Eds.), Aptitude, learning and instruction. Volume 3: Conative and affective process analyses (pp. 287-299). Hillsdale, NJ: Lawrence Erlbaum Associates.

Baumert, J. (1998). Learning math in school: Does interest really matter? In L. Hoffman, A. Krapp, K.A. Renninger, \& J. Baumert (Eds.), Interest and learning. Proceedings of the second conference on interest and gender (pp. 327-336). Kiel, Germany: IPN.

Chen, A. (1996). Student interest in a activities in a secondary physical education curriculum. An analysis of student subjectivity. Research Quarterly for Exercise and Sport, 67, 424-432.

Chen, A. (2001). A theoretical conceptualization for motivation research in physical education: an integrated perspective. Quest, 53, 35-58.

Chen, A., \& Darst, P-W. (2001). Situational interest in physical education: A function of learning task design. Research Quarterly for Exercise and Sport, 72, 150-164. 


\section{eJRIEPS 31 janvier 2014}

Chen, A., \& Darst, P-W. (2002). Individual and situational interest: The role of gender and skill. Contempory Educational Psychology, 27, 250-269.

Chen, A., \& Ennis, C.D. (2004). Searching for optimal motivators: Goals, interest, and learning in physical education. The Journal of Educational Research, 97, 329-338.

Chen, A., \& Ennis, C.D. (2009). Motivation and achievement in physical education. In K.R. Wentzler, \& A. Wigfield (Eds.), Handbook of Motivation at School (pp. 553-574). New-York, NY: Routledge.

Chen, A., \& Hancock, G.R. (2006). Conceptualizing a theoretical model for schoolcentered adolescent physical activity intervention research. Quest, 58, 355-376.

Chen, A., Darst, P.W., \& Pangrazi, P. (1999). What constitutes situational interest? Validating a construct in physical education. Measurement in Physical Education and Exercise Science, 3, 157-180.

Chen, A., Darst, P.W., \& Pangrazi, P. (2001). An examination of situational interest and its sources. British Journal of Educational Psychology, 71, 383-400.

Chen, A., Ennis, C-D., Martin, R., \& Sun, H. (2006). Situational interest: A curriculum component enhancing motivation to learn. In S.N. Hogan (Ed.), New developments in learning research (pp 235-261). New York, NJ: Nova Science Publishers.

Chen, A., Martin, R., Sun, H., \& Ennis, C. D. (2007). Is physical activity at risk in constructivist physical education? Research Quarterly for Exercise and Sport, 78, 500-509.

Chen, A., Shen, B., Tolley, C., \& Scrabis, K. (2002, April). Achievement goals, interests, and learning outcomes: A study on motivated learning in physical education. Paper presented at the annual meeting of the American Educational Research Association, New Orleans, LA.

Clifton, A. \& Gill, D.L. (1994). Gender differences in self-confidence on a feminine-typed task. Journal of Sport and Exercise Psychology, 16, 150-162.

Cosnefroy, L. (2004). Apprendre, faire mieux que les autres, éviter l'échec : l'influence de l'orientation des buts sur les apprentissages scolaires. Revue Française de Pédagogie, 147, 107-128.

Csikszentmihalyi, M. (1990). Flow: The psychology of optimal experience. New York: Harper \& Row.

Danner, F.W., \& Lonky, E. (1981). A cognitive-developmental approach to the effects of rewards on intrinsic motivation. Child Development, 52, 1043-1052. 


\section{eJRIEPS 31 janvier 2014}

Deci, E.L. (1992) The relation of interest to the motivation of behavior: A self-determination theory perpective. In K. A. Renninger. S. Hidi, \& A. Krapp (Eds.), The role of interest in learning and development (pp. 43-69). Hillsdale, NJ: Lawrence Erlbaum Associates.

Deci, E.L., \& Ryan, R.M. (1985). Intrinsic motivation and self-determination in human behavior. New York: Plenum.

Dewey, J. (1913). Interest and effort in education. Boston, MA: Riverside Press

Gardner, P.L. (1998). The development of males and females interest in science and technology. In P.L. Hoffman, A. Krapp, K.A. Renniger, \& J. Baumert (Eds.), Interest and learning. Proceedings of the second conference on interest and gender (pp. 41-57). Kiel, Germany: IPN.

Griffin, P.S. (1985). Teacher's perceptions of and responses to sex equity problems in middle school physical education program. Research Quarterly for Exercise and Sport, 56, 103-110.

Harackiewicz, J. M., Sansone, C., \& Manderlink, G. (1985). Competence, achievement, orientation, and intrinsic motivation. Journal of Personality and Social Psychology, 48, 493-508.

Harter, S. (1978). Pleasure derived from optimal challenge and the effects of extrinsic rewards on childrens's difficulty level choices. Child Development, 49, 788-799.

Hidi, Z. (2001). Interest, and learning: theoretical and practical considerations. Educational Psychology Review, 13, 191-209.

Hidi, S., \& Anderson, V. (1992). Situational interest and its impact on reading and expository writing. In K.A. Renninger, S. Hidi, \& A. Krapp (Eds.), The role of interest in learning and development (pp. 215-238). Hillsdale, NJ: Lawrence Erlbaum Associates.

Hidi, S., \& Baird, W. (1988). Strategies for increasing text-based interest and students' recall of expository texts. Reading Research Quarterly, 23, 465-483.

Hidi, S. \& Berndorff, D. (1998). Situational interest and learning. In L. Hoffmann, A. Krapp, K.A. Renninger, \& J. Baumerts (Eds.), Interest and learning. Proceedings of the second conference on interest and gender (pp. 74-90). Kiel, Germany: IPN.

Hidi, S., \& Harackiewicz, J.H. (2000). Motivating the academically unmotivated: A critical issue for the $21^{\text {st }}$ century. Review of Educational Research, 70, 151-179. 


\section{eJRIEPS 31 janvier 2014}

Krapp, A., Hidi, S. \& Renninger, K.A. (1992). Interest, learning, and development. In K.A. Renninger, S. Hidi, \& A. Krapp (Eds.), The role of interest in learning and development (pp. 1-26). Hillsdale, NJ: Lawrence Erlbaum Associates.

Lee, A.M., Fredenburg, K., Belcher, D., \& Cleveland, N. (1999). Gender differences in children's conceptions of competence and motivation in physical education. Sport, Education and Society, 4, 161-175.

Mitchell, M. (1993). Situational interest: Its multifaceted structure in the secondary school mathematics classroom. Journal of Educational Psychology, 85, 424-436.

Murphy, P.K., \& Alexander, P. (2000). A motivated exploration of motivation terminology. Contemporary Educational Psychology, 25, 3-53.

Reeve, J. (1996). Motivating others: Nurturing inner motivational resources. Needham Heights, MA: Allyn \& Bacon.

Schielfele, U., Krapp, A., \& Winteler, A. (1992). Interest as a predicator of academic achievement: A meta-analysis of research. In K. A. Renninger, S. Hidi, \& A. Krapp (Eds.), The role of interest in learning and development (pp. 183-212). Hillsdale, $\mathrm{NJ}$ : Lawrence Erlbaum Associates.

Shen, B., \& Chen, A. (2006). Examining the interrelations among knowledge, interests, and learning strategies. Journal of Teaching in Physical Education, 25, 182-199.

Shen, B., \& Chen, A. (2007). An Examination of Learning Profiles in Physical Education. Journal of Teaching in Physical Education, 26, 145-160.

Shen, B., Chen, A., \& Guan, J. (2007). Using achievement goals and interest to predict learning in physical education. The Journal of Experimental Education, 75, 89-108.

Shen, B., Chen A., Tolley, H., \& Scrabis, K.A. (2003). Gender and Interest-Based Motivation in learning Dance. Journal of Teaching in Physical Education, 22, 396409.

Spielberger, C.D., \& Starr, L. M. (1994). Curiosity and exploratory behavior. In H.F. O'Neil, Jr, \& M. Drillings (Eds.), Motivation: Theory and research (pp. 221-243). Hillsdale, NJ: Lawrence Erlbaum Associates.

Subramaniam, P.R. (2009). Motivational effects of interest on student engagement and learning in physical education: a review. International Journal of Physical Education, 46, 11-19.

Sun, H., Chen, A., Ennis, C., Martin, R., \& Shen, B. (2008). An examination of the multidimensionality of situational interest in elementary school physical education. Research Quarterly for Exercice and Sport, 79, 62-70. 
eJRIEPS 31 janvier 2014

Tobias, S. (1994). Interest, prior knowledge, and learning. Review of Educational Research, 64, 37-54.

Wentzler, K.R., \& Wigfield, A. (2009). Handbook of Motivation at School. New-York, NY : Rouledge.

Zhu, X., Chen, A., Ennis, C., Sun, H., Hopple C., Bonello M., Bae M., \& Kim S. (2009). Situational interest, cognitive engagement, and learning achievement in physical education. Contempory Educational Psychology, 34, 221-229. 